\section{(1) \\ CrossMark}

\title{
A review of psychosocial factors and personality in the treatment of obstructive sleep apnoea
}

\author{
Elizabeth A. Cayanan 1,2,3, Delwyn J. Bartlett ${ }^{1,4}$, Julia L. Chapman ${ }^{1,2}$, \\ Camilla M. Hoyos ${ }^{1,5,6}$, Craig L. Phillips ${ }^{1,4,7}$ and Ronald R. Grunstein ${ }^{1,2,4,7}$ \\ Number 4 in the Series "Sleep Disordered Breathing" \\ Edited by Renata Riha and Maria Bonsignore
}

Affiliations: ${ }^{1}$ CIRUS, Centre for Sleep and Chronobiology, Woolcock Institute of Medical Research, The University of Sydney, Sydney, Australia. ${ }^{2}$ NeuroSleep, National Health and Medical Research Council Centre of Research Excellence, Sydney, Australia. ${ }^{3}$ Faculty of Medicine and Health, Susan Wakil School of Nursing and Midwifery, The University of Sydney, Sydney, Australia. ${ }^{4}$ Faculty of Medicine and Health, Sydney Medical School, The University of Sydney, Sydney, Australia. ${ }^{5}$ Faculty of Science, School of Psychology, The University of Sydney, Sydney, Australia. ${ }^{6} \mathrm{Healthy}$ Brain Ageing Program, Brain and Mind Centre, Charles Perkins Centre, University of Sydney, Sydney, Australia. ${ }^{7}$ Sydney Health Partners (Royal North Shore and Royal Prince Alfred Hospitals), Sydney, Australia.

Correspondence: Elizabeth Cayanan, The Woolcock Institute of Medical Research, The University of Sydney, PO Box M77, Missenden Road, Sydney, NSW 2050, Australia. E-mail: elizabeth.cayanandsydney.edu.au

@ERSpublications

Personality traits and self-efficacy requires assessment prior to commencing CPAP or weight loss therapy in the management of OSA. These important factors may enhance treatment adherence and require ongoing assessment. bit.ly/2Q5TV19

Cite this article as: Cayanan EA, Bartlett DJ, Chapman JL, et al. A review of psychosocial factors and personality in the treatment of obstructive sleep apnoea. Eur Respir Rev 2019; 28: 190005 [https://doi.org/ 10.1183/16000617.0005-2019].

ABSTRACT Effective treatment of obstructive sleep apnoea (OSA) is primarily determined by adherence to the selected intervention. The most common treatment pathways are mechanical devices such as continuous positive airway pressure (CPAP) or a mandibular advancement device, often combined with weight loss therapy. Weight reduction is usually an adjunct therapy but may be used as a secondary treatment in mild-to-moderate OSA when mechanical treatments cannot be tolerated. To enhance the uptake and adherence to treatment, clinicians may assess patient's personality profiles and psychological readiness. There is a paucity of evidence related to these aspects of patient care and this article outlines the current research in relation to patient presentation, treatment uptake and barriers, and methods to enhance treatment adherence. This article disseminates personality traits observed in patients with OSA and identifies vulnerable groups who may require additional support to increase treatment adherence. It summarises the current evidence for treatment barriers in patients with OSA. Low self-efficacy in relation to CPAP and weight loss adherence will be explored as well as the potential to predict treatment responders and enhance therapeutic uptake and adherence. Extending personality traits into research and clinical practice could potentially result in more successful CPAP therapy and weight loss treatment outcomes.

Previous articles in this series: No. 1: Masa JF, Pepin J-L, Borel J-C, et al. Obesity hypoventilation syndrome. Eur Respir Rev 2019; 28: 180097. No. 2: Bruyneel M. Telemedicine in the diagnosis and treatment of sleep apnoea. Eur Respir Rev 2019; 28: 180093. No. : Ryan S, Arnaud A, Fitzpatrick SF, et al. Adipose tissue as a key player in obstructive sleep apnoea. Eur Respir Rev 2019; 28: 190006.

Provenance: Submitted article, peer reviewed.

Received: Jan 182019 | Accepted after revision: May 092019

Copyright $\odot$ ERS 2019. This article is open access and distributed under the terms of the Creative Commons Attribution Non-Commercial Licence 4.0. 


\section{Introduction}

Effective treatment of obstructive sleep apnoea (OSA) is primarily determined by adherence to one of three selected interventions of continuous positive airway pressure (CPAP), a mandibular advancement device (MAD) and/or weight loss therapy. Enhancing uptake and adherence to treatment is a key factor in clinical practice and there is limited research on the impact of patient personality in this process. This article explores personality traits in patients with OSA, where at present there is a paucity of specific research in this area. We aim to examine personality as an integral factor, along with a consideration of psychosocial factors, to improve the uptake and adherence to current OSA treatments. This article outlines the personality traits observed in patients with OSA and common methods of assessment, describes vulnerable groups including patients diagnosed with comorbid depression or type-D personality, and outlines treatment predictors and adherence, including the role of apathy in this condition. Finally, a clinical perspective will be presented in the context of this article.

\section{An overview of personality traits observed in patients with OSA}

Personality is a complex multidimensional system [1] relating to unconditional behaviour tendencies which then impact on how the individual functions in different circumstances [2]. The personality traits observed in OSA potentially correlate with the symptoms of the disorder, including chronic excessive daytime sleepiness [3]. When coupled with neurocognitive manifestations of OSA, which include impairments in vigilance, concentration, memory and executive function [4], the separation of personality and physiological OSA symptoms becomes increasingly difficult.

\section{Assessment of personality}

Objective assessment of these traits in the past has been detailed with multidimensional instruments such as the Minnesota multiphasic personality inventory (MMPI). However, the MMPI is a long (taking 1-2 h) and challenging assessment tool consisting of between 338 and 567 true/false items depending on the version of the publication used [5]. Any missing data require a mathematical adjustment between the missing items and the scale, which would complicate routine clinical assessment in patients with OSA [6]. Another tool that could be considered for the assessment of personality is the temperament and character inventory; however, there is no evidence suggesting patients with OSA have significantly elevated components of cooperativeness or self-directedness [7], which were identified as key personality components by Cloninger et al. [8] and Dell'Orco et al. [9]. This assessment tool consists of 240 items and can take up to 90 min to complete, making it challenging to conduct in routine clinical assessment [8]. There are many other personality assessment tools including the Swedish University's Scales of Personality $[10,11]$ and the revised NEO personality inventory [12]; however, none of these appear to have been validated in a sleep disordered breathing population and, therefore, we have not explored them in this article.

\section{Personality traits in patients with OSA}

Previous studies examining the relationship between the MMPI profiles and OSA have demonstrated discrepancies in psychiatric symptoms, personality and psychological features, and observed psychological difficulties. Comparisons between snorers and patients with OSA found significantly higher absolute scores and increased rates of clinical depression and hypochondriasis among individuals with OSA [13]. Patients with OSA had more inactivity, anergia, guilt, pessimism and low self-esteem, accompanied by prominent somatic concerns compared with snorers [13]. Hypochondriasis and psychopathic deviance were also significantly higher in a cross-sectional survey of 94 treatment-naïve patients with OSA compared with nonsnorers [14]. This greater overall psychopathology and the elevation on the hypochondriasis scale indicates somatic concerns and perhaps some antisocial attitudes in OSA [14]. In a larger study, 58\% of patients with OSA had at least one elevated MMPI score and the highest values were for depression, hypochondriasis and hysteria (an awareness of problems and vulnerabilities) [15]. Furthermore, more severe oxygen desaturation $(<92 \%)$ was associated with a higher number of elevations on the MMPI.

These elevated hypochondriasis scores may be associated with patients demonstrating extreme vigilance of physical symptoms, chronic fatigue, weakness and vague symptoms. The severity of psychopathology symptoms may also be associated with poor quality of life, attention deficit and subjective daytime sleepiness [14]. Individuals with psychopathic deviance may have difficulty adhering to rules, be reluctant to accept the advice of authority figures and fail to learn from previous mistakes. These personality traits of a somatic-neurotic type may amplify nonadherence with treatment therapies in OSA. Compared with narcoleptic patients, untreated patients with OSA exhibited more denial, hypochondriasis and hysterical personality characteristics, with lower anxiety and social introversion symptoms [16]. In the absence of supporting historical and observational data, the findings from the MMPI may be directly attributable to sleepiness that is secondary to OSA rather than reflective of personality disturbances in the traditional 
sense of psychiatric diagnoses [15]. Regardless, these influences present challenges in the uptake and maintenance of the use of various OSA therapies. Appropriate treatment of OSA alone may not be sufficient to improve patient quality of life. The maintenance of maladaptive hypochondriacal states in OSA negatively affect coping behaviours [14].

\section{Treatment of OSA}

The two treatment options most widely recommended for OSA are CPAP and/or weight loss. Weight reduction is usually an adjunct therapy but may be used as a secondary treatment in mild-to-moderate OSA when mechanical treatments cannot be tolerated. A MAD offers an additional treatment option; however, there is no evidence for this mode of treatment in relation to personality. This article will outline the evidence in relation to personality for adherence to each treatment option, suggest ways to enhance this adherence and look at potential alternative methods and therapies to address psychological and personality-related limitations.

\section{Barriers against and adherence to CPAP}

CPAP therapy is often considered the gold standard treatment for OSA, but adherence is poor with $~ 50 \%$ of patients being unable to use CPAP [17-19]. Biomedical factors have often been considered a barrier to treatment adherence; these factors include skin irritation, nose stuffiness, air leaks around the mask, claustrophobic reactions to the mask, problems with spontaneous intimacy with the bed partner and noise of the machine [19]. Although these factors have been reduced by improvements in machine design, the objective treatment adherence remains limited. Psychological models have been instituted in the prediction of CPAP acceptance and subsequent adherence [20-25]. Patients have formed beliefs and expectations about the treatment prior to commencing any therapy which influences their subjective experience. Adherence early in the treatment continuum is one of the strongest predictors of subsequent use compared with the biomedical indices of disease severity [21, 26, 27].

The presence of depression may influence treatment adherence with CPAP therapy in patients with OSA [28]. Questionnaire-based assessment of self-reported CPAP therapy adherence showed an association between depression and lower CPAP use [29], while an objective assessment of newly prescribed users found no association between CPAP therapy adherence and depression 1 month after treatment commenced [30]. Several other studies have published inconsistent findings in relation to depression and CPAP therapy adherence $[24,31-36]$. However, the treatment of depression also needs to be considered as a potential means of alleviating the therapeutic burden of CPAP and enhancing treatment uptake and maintenance.

Other factors such as lower socioeconomic status are important considerations, where patients in this group were less receptive to CPAP treatment than those of a higher status [37]. Patients with OSA and poorer socioeconomic status have higher rates of obesity, glucose intolerance and cigarette smoking, and poorer adherence to CPAP, all contributing factors in cardiovascular disease and mortality [38-41]. Poor socioeconomic status is a factor in many health interventions, impairing treatment acceptance, including CPAP treatment [37]. While these barriers are a broad-reaching issue in a clinical setting, additional education and support may need to be directed to these patients upon treatment initiation.

\section{Groups who are vulnerable to nonadherence in the treatment of OSA Depression and OSA}

The mechanisms underlying the association between OSA and depressive symptoms are unclear. Plausible explanations include poor sleep quality, frequent arousals and intermittent hypoxaemia affecting mood $[42,43]$. Physiological explanations include the release of several proinflammatory cytokines $[44,45]$ or neurotransmitter involvement in sleep/wake states [46]. Frequent comorbid chronic conditions such as obesity, diabetes and cardiovascular disease act as potential causes/triggers of depression [46]. Cross-sectional studies generally show a higher prevalence of depression among patients with OSA in both community and sleep disorder clinic samples [28,47]. This relationship is complicated by overlapping symptoms and, therefore, it is essential to consider the interaction between these disorders. Evidence related to the change in depression scores following treatment of OSA compared with the general population is mixed, suggesting a more complex relationship [48]. CPAP therapy appears to ameliorate some of the symptoms of OSA with a flow on improvement in quality of life and depressive symptoms [49-55]. A direct causal relationship would mean depression is expected to improve consistently with effective OSA treatment. Few studies have explored the effects of CPAP therapy on depression in patients with OSA (or have failed to use appropriate diagnostic tools) [28]. A recent systematic review and meta-analysis of 19 randomised controlled studies evaluated the efficacy of CPAP or a MAD and found significant improvement in depression compared with control groups [24]. In contrast, a Cochrane meta-analysis found that CPAP improved depression but when a random effects model was used it was no 
longer significant [56]. The overall evidence suggests CPAP therapy may be effective in improving mood states in patients with severe depressive symptoms that are secondary to their OSA [57].

\section{Type-D personality and physiological correlates}

Type- $\mathrm{D}$ personality is defined as a combination of high negative affect and high social isolation and is associated with poor health outcomes [58]. These combined factors increase vulnerability for impaired physical and mental health status in various patient groups [59-61]. The type-D construct is measured with the short and simple DS14 questionnaire [62,63]. There are two seven-item subscales assessing negative affectivity (e.g. "I often feel unhappy") and social inhibition (e.g. "I am a closed person") [64]. Individuals are categorised as type-D using a standardised cut-off score $\geqslant 10$ on both the negative affectivity and social inhibition subscales.

The pathophysiological mechanisms driving type-D personality towards adverse cardiovascular outcomes are largely unknown [65]. In a population-based cohort study, type-D was associated with fewer sleeping hours and an increased risk of sleep disturbance in adolescents [66]. Type-D personality has been associated with an increased systolic and diastolic blood pressure in response to the cold pressor test as a marker of autonomic nervous system dysfunction [67]. Interestingly, recent research suggests the interplay between autonomic nervous system dysfunction and inflammation contribute to the cardiovascular disease-related mortality risk associated with depression [68].

Higher HbAlc and fasting plasma glucose are also different in type-D personality, indicating poorer glycaemic control, along with higher fibrinogen and lower night-time heart rate variability [65]. Systemic inflammation and poorer autonomic nervous system modulation appear to be underlying explanatory factors [65]. These patients subjectively reported less social support and more sleep difficulties [69], which were independent of lifestyle factors (alcohol, cigarette consumption and physical activity) and increased body mass index was not significant [65]. Elevated plasma glucose was also found in a longitudinal occupational cohort of patients without OSA who were classified as type-D compared with their counterparts [70]. Type-D personality has a significant negative impact on cardio-metabolic health, which may relate to patients with untreated OSA.

To date there have been no studies that have assessed the long-term health outcomes of patients with type-D personality with OSA. One study assessed the association between type-D personality and sleep quality using objective polysomnography and the subjective Pittsburgh Sleep Quality Index [71]. In patients with comorbid OSA and cardiac disease, type-D was more prevalent in men with OSA compared to those without $(32.5 \%$ versus $25.4 \%, \mathrm{p}<0.04)$ [71]. Those with type-D personality reported more complaints related to subjective sleep quality, irrespective of their OSA diagnosis compared with non-type-D personality. In the general population, individuals with type- $\mathrm{D}$ personality have a heightened perception of negative emotions such as depression and anxiety, potentially contributing to a bi-directional relationship between personality and sleep, with adverse health outcomes [64]. The association with negative health outcomes coupled with impaired cognitive function [72] and decreased quality of life [73], necessitate identification of type-D personality prior to commencing treatment and ideally modification of these factors where possible, to enhance health outcomes.

\section{Predicting CPAP adherence}

Clinicians are confronted with an effective treatment option (CPAP) which has a low adherence rate. The issues surrounding CPAP adherence are complicated and include physician, patient and healthcare system influences, and no single factor is consistently predictive of CPAP acceptance and adherence. Personality plays a role in CPAP adherence, and therefore should be assessed and addressed prior to initiating treatment.

Two primary theories of behaviour change have been applied to CPAP adherence: the transtheoretical model (TTM) [74] and the social cognitive theory (SCT) [75]. The TTM proposes that people fall on a continuum of motivational readiness to change their current behaviour; ranging from pre-contemplative to maintenance. Moving through this continuum involves decisional balance, whereby the potential gains and losses of engaging in a new behaviour are considered. Once readiness to change is established, the perceived gains of changing behaviour usually outweigh the losses. The SCT is more applicable to those who are ready to change and focuses on problem solving, coping skills, goal setting, reinforcement, self-efficacy and outcome expectations. Self-efficacy is especially relevant in the initiation of behaviour change, requiring self-reflection, effort and perseverance in adverse situations [75]. Controversy remains regarding the most appropriate time to assess these constructs. STEPNOWSKY et al. [20] used OSA-specific scales of TTM and the self-efficacy construct within the SCT in acute and 1-month CPAP adherence assessments. These constructs accounted for more of the variance in CPAP adherence at both time-points than subjective sleepiness or CPAP pressure. Some groups suggest CPAP therapy should be used for 
1 week before self-efficacy can be determined; however, a randomised controlled trial found that self-efficacy measured prior to the commencement of CPAP predicted adherence [76]. Readiness and self-efficacy can be measured prior to treatment initiation. These principles of behaviour change are modifiable and when identified as low, may improve with additional education and practical support early in the treatment process.

A latent profile analysis has been used to identify CPAP user profiles [77]. Adherence was predicted by self-efficacy, insomnia, apnoea-hypopnoea index, time since CPAP was initiated and CPAP pressure. Social cognitive variables were measured through the self-efficacy measure for sleep apnoea, a 26-item questionnaire assessing CPAP adherence-related cognition based on the SCT. The self-efficacy measure for sleep apnoea proved useful in identifying those who need additional support at diagnosis to enhance treatment adherence.

The health belief model has been proposed as a conceptual basis for understanding patient motivations to accept and, subsequently, adhere to CPAP treatment $[26,78]$. This model proposed that readiness to act hinges on individual risk perception (susceptibility to illness or consequences if left untreated) and the perceived significance of their illness (impact on current functioning) [26]. The patient's belief in the benefit of CPAP treatment requires weighing the perceived benefits to their health (outcome expectancies) against the perceived barriers to using CPAP (potential side-effects of the treatment). The patient's self-efficacy in being able to use the treatment despite barriers, as well as the presence of a cue to action (such as advice from medical professionals, encouragement from their spouse, or media campaigns) are also important in the model $[78,79]$.

The constructs of beliefs and barriers have been shown to be better predictors of CPAP adherence compared with objective measures of disease severity [80]. The predictive potential could be applied after diagnosis and prior to initiation of CPAP therapy or after just one night of CPAP use, thus posing a clinically feasible adherence outcome model [26]. Patients' outcome expectations prior to using CPAP and perception of risk and functional limitations due to sleepiness (such as intimacy, activity levels and general productivity) all uniquely predicted CPAP initiation and adherence [26]. Patients who were predicted to have low adherence can be identified and supported appropriately using these predictive models.

In addition to the TTM, SCT and health belief model, the behavioural inhibition system/behavioural activation system (BIS/BAS) model of personality has been proposed to describe CPAP adherence [81]. BAS regulates desirable motives, the goal being to move towards something desired that produces joy; and the BIS regulates aversive motives, the goal being to move away from something aversive that produces fear and anxiety [82]. It is proposed that people are more sensitive to either BAS or BIS. The positive outcome of reduced sleepiness and cardiovascular risk appeals to BAS-sensitive patients while BIS-sensitive patients will be more likely not to adhere to CPAP because of negative feelings and anxiety (due to the socially undesirable attributes of the invasive CPAP mask and machine) [81]. This supports the concept of the influence of type-D personality, whereby individuals who have a negative affect and social inhibition, are less likely to adhere to CPAP. After 1 month of CPAP, nonadherence was associated with elevated BIS scores and neuroticism [81]. In contrast, a larger more recent study in 321 US veterans showed affect, intellect and fun-seeking behaviours correlated with CPAP adherence [83]. Certainly, type-D personality character traits do not appear to favour positive treatment outcomes. Identification of these personality types prior to treatment allocation and cognitive behavioural therapy (CBT) may improve the treatment outcome in these cases.

Factors affecting individual's intention to use CPAP and their actual decision to commence CPAP treatment are important in examining a relationship between behavioural intention and actual action [84]. In the study, the health belief model was used and the predominant driving factors appeared to be around patient awareness of behavioural intention which enabled decision-makers to promote treatment when developing targeted interventions. Again, patients' attitudes and health beliefs prior to OSA diagnosis predict the intention to be treated for OSA and, in turn, affect the actual decision to commence treatment $[84]$.

In a recent comprehensive review [85], the impact of type-D personality on CPAP adherence was associated with poorer adherence and treatment outcomes due to negative affectivity, social inhibition, unhealthy lifestyle, and a reluctance to consult and/or follow medical advice [85]. Prevalence of type-D personality in 247 patients with OSA using treatment for $>6$ months was $30 \%$, with a significantly lower objective adherence to CPAP therapy and more frequent and severe side-effects reported [86]. In a replication of this study in 265 participants, no significant association was found between CPAP adherence and type-D personality; however, depression was associated with poor compliance [87]. Patients more likely to adhere to CPAP therapy demonstrated a high internal locus of control, high self-efficacy, were self-referred for treatment and had active coping skills [85]. A conservative approach suggests that 
personality and depression should still be considered as potential predictors of CPAP compliance that require attention when measured as being compromised to ensure the appropriate interventions are applied.

\section{Enhancing therapeutic adherence}

The ultimate goal of optimising treatment adherence can be achieved through early prediction of treatment adherence followed by the delivery of targeted adherence interventions. Using an index which identified CPAP non-adherers immediately after titration, polysomnography allowed for targeted interventions to promote adherence [88]. Education and support programmes increase patients' acceptance of CPAP therapy during treatment initiation with a focus on improving patient symptoms and perception of therapy [17, 89-91].

CPAP treatment can progressively decrease the psychopathological signs of OSA, described earlier in this article alongside a generalised improvement in psychosocial adaptation. Patient satisfaction with CPAP treatment positively correlates with adherence, with the greater day-to-day improvement resulting in a greater likelihood of using CPAP therapy [59]. Severe OSA is associated with serious psychosocial dysfunction that gradually improves with CPAP therapy [92]. Therefore, if a patient is identified with unfavourable personality traits (personality type-D or a tendency towards the BIS or low readiness or self-efficacy) this needs to be addressed prior to initiating therapy. This early identification of potential difficulties is important to enhance treatment adherence while the maintenance of CPAP therapy will potentially enhance positive psychosocial wellbeing. Behavioural therapies have been proposed as a potential means of enhancing CPAP adherence in order to address these traits [93-95]. These include, but are not limited to, both conservative and more intensive methods. Conservative interventions include educational pamphlets [96], single group educational sessions [97] and more intensive therapies such as group CBT [76], motivational enhancement interventions [98] or self-management programmes [99].

These conservative and traditional interventions may be adequate for enhancing CPAP adherence in some patients; however, others may need more time and resource-intensive CBT interventions [95]. One such proposal is a cost-effective alternative using adaptive interventions while conserving healthcare resources. These adaptive interventions or stepped-care models do not deliver all treatment components, instead the patient receives the initial treatment (such as a brief educational intervention) and those who do not respond are prescribed additional treatment (more intensive CBT). Patients are guided through individualised treatments that correspond to their specific needs and performance levels. This model is not one-size fits all and provides appropriate and targeted patient-centred care. This model was piloted in patients with OSA, with a more intensive phase of intervention (motivational enhancement/ self-management treatments) to those who did not demonstrate a $25 \%$ increase in therapeutic use following the conservative educational session [95]. The use of this adaptive model appeared feasible, acceptable and efficacious. There was an increased use of therapy alongside improved sleep quality and reduced daytime sleepiness and potentially could be extended to concurrent weight loss therapy in a clinical setting [100].

\section{The role of personality in weight loss therapy}

To our knowledge, there are no studies describing the psychosocial barriers to weight loss therapy specifically in patients with OSA, nor the potential role of personality. Therefore, this article briefly outlines the evidence for personality in general obesity in order to overlay relevant considerations in the context of OSA. In the general obesity literature, the concept of personality has been broadly explored in both randomised and observational studies. A recent review suggested a range of contributing factors that enhance weight loss maintenance, including behavioural factors (such as exercise, calorie control and self-monitoring), cognitive factors (such as reduced disinhibition, satisfaction with achieved results and confidence in self-management) alongside personality traits (such as low novelty seeking) [101]. Weight loss surgery outcomes have also been assessed in light of personality factors and a pattern for "self-regulation in spite of the urge or demands of the moment" emerged as a strong predictor of sustained weight loss outcomes across nine recent studies [102]. Previous examination of personality in this realm found that the presence of a personality disorder was the only preoperative psychosocial factor unequivocally associated with suboptimal weight loss outcomes [103] and interestingly, type-D personality has not been investigated as a barrier to weight loss. Another recent review of various methods of weight loss sought to assess personality traits, as measured by the temperament and character inventory and their association with treatment outcome. This review found novelty seeking and self-directedness traits to be predictors of short-term weight loss ( $\leqslant 6$ months) while persistence and novelty seeking traits may be related to long-term weight loss maintenance ( $\geqslant 12$ months) [104]. Novelty seeking refers to the tendency to seek out new stimuli and experiences, be easily bored, impulsive, inclined to avoid monotony and have a strong appetite [105]. Self-directedness is a character trait and measures concepts about oneself, 
self-acceptance and the ability to direct one's own life according to personal goals and values [104]. Persistence was defined as the preservation of resistance-to-frustration behaviour [104]. These personality traits differ from those associated with type-D and also those identified as being related to CPAP nonadherence. If these constructs are consistent in an OSA population, it would be important for these personality traits to also be assessed during OSA diagnosis to identify patients that may demonstrate barriers to adherence with weight loss therapy.

\section{Predicting weight loss success}

Health self-efficacy is the belief in one's capability to perform health behaviours. As in CPAP adherence, health self-efficacy is a significant factor in eliciting health behaviour change such as weight loss. It is useful to identify barriers and facilitators of weight loss success in OSA prior to investing in treatment, much like it is useful to predict barriers and facilitators of CPAP adherence prior to commencing treatment. Unlike CPAP therapy, the benefit of weight loss aids in the resolution of OSA disease rather than only treating the presenting symptoms. The challenge lies in maintaining the weight loss achieved. There is no evidence for predictors of weight loss treatment responders in OSA; however, attention has been given to exercise interventions in patients with OSA. Intention to exercise was assessed in a cross-sectional population of patients with OSA in the context of TTM of behaviour change and identified four patient profiles based on the perceived costs and benefits of exercise and exercise-related self-efficacy [106]. The results suggested it was possible to identify patients who were more likely to engage in more exercise, and to identify barriers to exercise in patients less inclined to increase their exercise, both of which provided opportunities to intervene and enhance patient uptake and adherence to interventions. Qualitative evidence extended this profiling and indicated that strong incentives to change seemed crucial for engagement in physical activity as a component of a weight loss intervention [107]. Similar to CPAP therapy initiation and adherence, exploration of the patient's expectations of behaviour change related to physical activity and the perceived facilitators and barriers for engagement may be helpful when tailoring behavioural support strategies [107].

Beyond physical activity, the construct of self-efficacy has been well documented in general obesity literature. Evidence suggests that pre-treatment diet and exercise self-efficacies can predict weight loss success and changes in diet self-efficacy throughout treatment appears to be a stronger predictor than baseline levels [108]. In addition to self-efficacy, a recent cross-sectional non-OSA community study conducted in the USA suggested that prior success with dietary self-regulation may predict better adherence to and success in behavioural weight control programmes [109].

It is well established that women are more likely to initiate weight loss treatment compared with men; however, OSA is more prevalent in men. This translates to the majority of the OSA population being comparatively less likely to access weight loss as a treatment for OSA. This is also seen in the rates of patients without OSA accessing bariatric surgery; whereby the evidence suggests that despite similar rates of obesity, women disproportionately make up the majority of the bariatric surgery population [110]. This barrier should be considered when recommending OSA treatment to ensure that men are adequately directed towards weight loss therapy and their adherence followed up in a clinical setting.

\section{Apathy and OSA: a barrier to treatment adherence}

Apathy is a symptom distinct from depression characterised by a loss of motivation, poor effort and initiation, and the term describes a lack of motivation, flat affect and loss of energy [111]. The prevalence of apathy in the obese population is estimated at $50 \%$ and appears to be evident in OSA [112]. Treating apathy may assist in adherence to weight management programmes and its role has been examined in the effectiveness of such programmes among veteran patients without OSA through pharmacotherapy (methylphenidate) that targeted apathy and is also known to promote wakefulness [113]. Modafinil is another type of pharmacotherapy that promotes wakefulness and has previously been trialled in the management of apathy in a sample of patients with Alzheimer's disease $[114,115]$ and may have some benefit in treating apathy in this disorder. Interestingly, the R-enantiomer of this medication (armodafinil) was trialled in an OSA cohort in combination with weight loss therapy for the treatment of daytime sleepiness (rather than apathy) and was found to enhance weight loss [116]. It has been postulated that the mechanism by which this occurs is the activation of the reward centres in the brain $[117,118]$. It therefore seems pertinent to consider the pharmacological management of apathy in patients with OSA prior to commencing weight loss therapy as a means of enhancing weight loss maintenance.

\section{Enhancing weight loss adherence}

The adaptive or stepped-care model of delivering behaviour change therapy that was explored in the context of CPAP is also relevant in the management of obesity in patients with OSA. The effectiveness of both conservative and more intensive weight loss programmes has been well documented in OSA [119-124]. The 
adaptive model proposes that conservative approaches should be initiated (such as information pamphlets or community referrals) and that nonresponders be referred to more intensive models, such as very low energy diets and/or psychological therapeutic models. This sequential, individualised approach whereby treatment is adapted and readapted over time in response to the specific needs and evolving status of the individual has been explored in general obesity [100]. This trial sought to provide an optimal adaptive model through the sequential multiple assignment randomised trial (SMART) and outlines a tailored approach to optimise weight loss [100]. This approach further justifies the benefit in pre-screening individuals to ascertain their readiness to change, their self-efficacy and their beliefs around planned treatment to ensure the approach taken is suitable for their personality and psychological readiness. In addition, evaluating previous successful weight control efforts also means that individuals can be better matched to those treatments likely to yield success with weight loss and those requiring additional support can be identified [109].

In parallel with adaptive care models, other aspects have been targeted through interventions for the purpose of improving weight loss outcomes. For example, the enhancement of self-efficacy through individual sessions in addition to standard weight loss treatment among female patients without OSA was shown to improve weight loss maintenance [125]. A novel approach to improving self-efficacy in overweight patients without OSA was recently evaluated through a randomised controlled trial using virtual embodiment and play [126]. This social virtual world (Second Life) was designed to increase exercise and nutrition efficacy and the findings supported the use of a virtual world and gaming to improve exercise efficacy and weight loss and may be relevant to an OSA population.

Regardless of the methodology or approach undertaken, the benefit of weight loss in patients with OSA far outweighs not addressing comorbid obesity. Future research should further investigate opportunities to identify treatment responders or describe phenotypes that are more responsive to weight loss interventions. Personality, self-efficacy and behaviour should be better characterised in this population to enhance weight loss success. Adaptive interventions in conjunction with novel approaches including virtual reality and mobile phone or computer applications should be explored in OSA.

\section{Clinical summary and conclusion}

Patients with OSA have more inactivity, anergia, guilt, pessimism and low self-esteem and there is also evidence for the persistence of type-D personality in this population. This is accompanied by prominent somatic concerns and significantly higher rates of clinically elevated hypochondriasis and psychopathic deviance. These personality traits may be secondary to OSA rather than reflective of personality disturbances in the traditional sense; however, they present challenges in the uptake and maintenance of the use of mechanical devices or weight loss therapy. Those patients with more severe oxygen desaturation may be more vulnerable to the psychopathologies mentioned above. From a clinical perspective, the key to successful treatment lies in measuring a patient's readiness for change and, if necessary, targeting their self-efficacy and making accommodation for any personality traits which would inhibit treatment uptake and adherence. Phenotyping personality and beliefs is applicable both to mechanical therapies and weight loss therapy. There has been little exploration of personality in the context of weight loss for OSA and future research needs to expand this important area for vulnerable individuals, with the primary goal of improving treatment outcomes.

Conflict of interest: None declared.

Support statement: E.A. Cayanan and J.L. Chapman receive funding through the National Health and Medical Research Council Centre of Research Excellence (NeuroSleep Sydney, NSW, Australia). R.R. Grunstein receives funding through a National Health and Medical Research Council Senior Principal Research Fellowship. C.L. Phillips receives funding through a National Health and Medical Research Council Boosting Dementia Research Leadership Fellowship and the Sydney Medical School Foundation Chapman Fellowship. C.M. Hoyos receives funding through a National Health and Medical Research Council - Australian Research Council Dementia Research Development Fellowship. Funding information for this article has been deposited with the Crossref Funder Registry.

\section{References}

1 Caprara GV, Vecchione M, Alessandri G, et al. The contribution of personality traits and self-efficacy beliefs to academic achievement: a longitudinal study. Br J Educ Psychol 2011; 81: 78-96.

2 McCrae RR Jr, Costa PT. A five-factor theory of personality. In: Pervin LA, John OP, eds. Handbook of personality: theory and research. 2nd Edn. 1999; pp. 139-153.

3 Sateia MJ. Neuropsychological impairment and quality of life in obstructive sleep apnea. Clin Chest Med 2003; 24: 249-259.

4 Day R, Gerhardstein R, Lumley A, et al. The behavioral morbidity of obstructive sleep apnea. Prog Cardiovasc Dis 1999; 41: 341-354. 

135-136.

6 Gotts EE, Knudsen TE. The Clinical Interpretation of MMPI-2: a Content Cluster Approach. Abingdon, Routledge, 2005.

7 Sforza E, de Saint Hilaire Z, Pelissolo A, et al. Personality, anxiety and mood traits in patients with sleep-related breathing disorders: effect of reduced daytime alertness. Sleep Med 2002; 3: 139-145.

8 Cloninger C, Svrakic D, Przybeck T. A psychobiological model of temperament and character. Arch Gen Psychiatry 1993; 50: 975-990.

9 Dell'Orco S, Sperandeo R, Moretto E, et al. Revision on psychometric properties of the temperament and character inventory in a clinical sample. Front Psychol 2018; 9: 1951.

10 Gustavsson JP, Bergman H, Edman G, et al. Swedish universities scales of personality (SSP): construction, internal consistency and normative data. Acta Psychiatr Scand 2000; 102: 217-225.

11 Barrett PT, Petrides KV, Eysenck SB, et al. The Eysenck personality questionnaire: an examination of the factorial similarity of P, E, N, and L across 34 countries. Pers Indiv Differ 1998; 25: 805-819.

12 Costa PT Jr, McCrae RR. Stability and change in personality assessment: the revised NEO personality inventory in the year 2000. J Pers Assess 1997; 68: 86-94.

13 Aikens JE, Mendelson WB. A matched comparison of MMPI responses in patients with primary snoring or obstructive sleep apnea. Sleep 1999; 22: 355-359.

14 Ekici A, Ekici M, Oguzturk O, et al. Personality profiles in patients with obstructive sleep apnea. Sleep Breath 2013; 17: 305-310.

15 Aikens JE, Caruana-Montaldo B, Vanable PA, et al. MMPI correlates of sleep and respiratory disturbance in obstructive sleep apnea. Sleep 1999; 22: 362-369.

16 Beutler LE, Ware JC, Karacan I, et al. Differentiating psychological characteristics of patients with sleep apnea and narcolepsy. Sleep 1981; 4: 39-47.

17 Weaver TE, Grunstein RR. Adherence to continuous positive airway pressure therapy: the challenge to effective treatment. Proc Am Thorac Soc 2008; 5: 173-178.

18 Collard P, Pieters T, Aubert G, et al. Compliance with nasal CPAP in obstructive sleep apnea patients. Sleep Med Rev 1997; 1: 33-44.

19 Zozula R, Rosen R. Compliance with continuous positive airway pressure therapy: assessing and improving treatment outcomes. Curr Opin Pulm Med 2001; 7: 391-398.

20 Stepnowsky CJ Jr, Marler MR, Ancoli-Israel S. Determinants of nasal CPAP compliance. Sleep Med 2002; 3: 239-247.

21 Aloia MS, Arnedt JT, Stepnowsky C, et al. Predicting treatment adherence in obstructive sleep apnea using principles of behavior change. J Clin Sleep Med 2005; 1: 346-353.

22 Edinger JD, Carwile S, Miller P, et al. Psychological status, syndromatic measures, and compliance with nasal CPAP therapy for sleep apnea. Percept Mot Skills 1994; 78: 1116-1118.

23 McFadyen TA, Espie CA, McArdle N, et al. Controlled, prospective trial of psychosocial function before and after continuous positive airway pressure therapy. Eur Respir J 2001; 18: 996-1002.

24 Stepnowsky CJ Jr, Bardwell WA, Moore PJ, et al. Psychologic correlates of compliance with continuous positive airway pressure. Sleep 2002; 25: 758-762.

25 Wild MR, Engleman HM, Douglas NJ, et al. Can psychological factors help us to determine adherence to CPAP? A prospective study. Eur Respir J 2004; 24: 461-465.

26 Olsen S, Smith S, Oei TP. Adherence to continuous positive airway pressure therapy in obstructive sleep apnoea sufferers: a theoretical approach to treatment adherence and intervention. Clin Psychol Rev 2008; 28: 1355-1371.

27 Engleman HM, Wild MR. Improving CPAP use by patients with the sleep apnoea/hypopnoea syndrome (SAHS). Sleep Med Rev 2003; 7: 81-99.

28 BaHammam AS, Kendzerska T, Gupta R, et al. Comorbid depression in obstructive sleep apnea: an under-recognized association. Sleep Breath 2016; 20: 447-456.

29 Kjelsberg FN, Ruud EA, Stavem K. Predictors of symptoms of anxiety and depression in obstructive sleep apnea. Sleep Med 2005; 6: 341-346.

30 Poulet C, Veale D, Arnol N, et al. Psychological variables as predictors of adherence to treatment by continuous positive airway pressure. Sleep Med 2009; 10: 993-999.

31 Gagnadoux F, Le Vaillant M, Goupil F, et al. Depressive symptoms before and after long-term CPAP therapy in patients with sleep apnea. Chest 2014; 145: 1025-1031.

32 Lewis KE, Seale L, Bartle IE, et al. Early predictors of CPAP use for the treatment of obstructive sleep apnea. Sleep 2004; 27: 134-138.

33 Law M, Naughton M, Ho S, et al. Depression may reduce adherence during CPAP titration trial. J Clin Sleep Med 2014; 10: 163-169.

34 Wallace DM, Vargas SS, Schwartz SJ, et al. Determinants of continuous positive airway pressure adherence in a sleep clinic cohort of South Florida Hispanic veterans. Sleep Breath 2013; 17: 351-363.

35 Wallace DM, Wohlgemuth WK. Does race-ethnicity moderate the relationship between CPAP adherence and functional outcomes of sleep in US veterans with obstructive sleep apnea syndrome? J Clin Sleep Med 2014; 10: 1083-1091.

36 Means MK, Ulmer CS, Edinger JD. Ethnic differences in continuous positive airway pressure (CPAP) adherence in veterans with and without psychiatric disorders. Behav Sleep Med 2010; 8: 260-273.

37 Simon-Tuval T, Reuveni H, Greenberg-Dotan S, et al. Low socioeconomic status is a risk factor for CPAP acceptance among adult OSAS patients requiring treatment. Sleep 2009; 32: 545-552.

38 Jenkinson C, Davies RJ, Mullins R, et al. Comparison of therapeutic and subtherapeutic nasal continuous positive airway pressure for obstructive sleep apnoea: a randomised prospective parallel trial. Lancet 1999; 353 2100-2105.

39 Marin JM, Carrizo SJ, Vicente E, et al. Long-term cardiovascular outcomes in men with obstructive sleep apnoea-hypopnoea with or without treatment with continuous positive airway pressure: an observational study. Lancet 2005; 365: 1046-1053. 
Tarasiuk A, Greenberg-Dotan S, Simon T, et al. Low socioeconomic status is a risk factor for cardiovascular disease among adult obstructive sleep apnea syndrome patients requiring treatment. Chest 2006; 130: 766-773.

Mackenbach JP, Stirbu I, Roskam AJ, et al. Socioeconomic inequalities in health in 22 European countries. N Engl J Med 2008; 358: 2468-2481.

Chen YH, Keller JK, Kang JH, et al. Obstructive sleep apnea and the subsequent risk of depressive disorder: a population-based follow-up study. J Clin Sleep Med 2013; 9: 417-423.

Bardwell WA, Norman D, Ancoli-Israel S, et al. Effects of 2-week nocturnal oxygen supplementation and continuous positive airway pressure treatment on psychological symptoms in patients with obstructive sleep apnea: a randomized placebo-controlled study. Behav Sleep Med 2007; 5: 21-38.

Kasasbeh E, Chi DS, Krishnaswamy G. Inflammatory aspects of sleep apnea and their cardiovascular consequences. South Med J 2006; 99: 58-67.

Irwin MR, Miller AH. Depressive disorders and immunity: 20 years of progress and discovery. Brain Behav Immun 2007; 21: 374-383.

Katon W, Lin EH, Kroenke K. The association of depression and anxiety with medical symptom burden in patients with chronic medical illness. Gen Hosp Psychiatry 2007; 29: 147-155.

Bhatia M, Khatiwada S. Co-morbid OSA and depression: what we know and what we need to know. Sleep Vigilance 2017; 1: 7-11.

Haba-Rubio J. Psychiatric aspects of organic sleep disorders. Dialogues Clin Neurosci 2005; 7: 335-346. with nasal continuous positive airway pressure. J Clin Psychiatry 1989; 50: 348-351. Silva GE, An MW, Goodwin JL, et al. Longitudinal evaluation of sleep-disordered brea with change in quality of life: the sleep heart health study (SHHS). Sleep 2009; 32: 1049-1057.

Kawahara S, Akashiba T, Akahoshi T, et al. Nasal CPAP improves the quality of life and lessens the depressive symptoms in patients with obstructive sleep apnea syndrome. Intern Med 2005; 44: 422-427.

Engleman HM, Cheshire KE, Deary IJ, et al. Daytime sleepiness, cognitive performance and mood after continuous positive airway pressure for the sleep apnoea/hypopnoea syndrome. Thorax 1993; 48: 911-914.

Means MK, Lichstein KL, Edinger JD, et al. Changes in depressive symptoms after continuous positive airway pressure treatment for obstructive sleep apnea. Sleep Breath 2003; 7: 31-42.

Schwartz DJ, Kohler WC, Karatinos G. Symptoms of depression in individuals with obstructive sleep apnea may be amenable to treatment with continuous positive airway pressure. Chest 2005; 128: 1304-1309.

Diamanti C, Manali E, Ginieri-Coccossis M, et al. Depression, physical activity, energy consumption, and quality of life in OSA patients before and after CPAP treatment. Sleep Breath 2013; 17: 1159-1168.

Giles TL, Lasserson TJ, Smith BH, et al. Continuous positive airways pressure for obstructive sleep apnoea in adults. Cochrane Database Syst Rev 2006; 3: Cd001106.

Yu BH, Ancoli-Israel S, Dimsdale JE. Effect of CPAP treatment on mood states in patients with sleep apnea. $J$ Psychiatr Res 1999; 33: 427-432.

Denollet J, Pedersen SS, Ong AT, et al. Social inhibition modulates the effect of negative emotions on cardiac prognosis following percutaneous coronary intervention in the drug-eluting stent era. Eur Heart J 2006; 27: $171-177$.

Mols F, Denollet J. Type D personality among noncardiovascular patient populations: a systematic review. Gen Hosp Psychiatry 2010; 32: 66-72.

Dulfer K, Hazemeijer BA, Van Dijk MR, et al. Prognostic value of type D personality for 10-year mortality and subjective health status in patients treated with percutaneous coronary intervention. J Psychosom Res 2015; 79: 214-221.

Grande G, Romppel M, Barth J. Association between type D personality and prognosis in patients with cardiovascular diseases: a systematic review and meta-analysis. Ann Behav Med 2012; 43: 299-310.

Denollet J. DS14: standard assessment of negative affectivity, social inhibition, and Type D personality. Psychosom Med 2005; 67: 89-97.

Kupper N, Denollet J. Type D personality as a prognostic factor in heart disease: assessment and mediating mechanisms. J Pers Assess 2007; 89: 265-276.

Mols F, Denollet J. Type D personality in the general population: a systematic review of health status, mechanisms of disease, and work-related problems. Health Qual Life Outcomes 2010; 8: 9.

Jandackova VK, Koenig J, Jarczok MN, et al. Potential biological pathways linking Type-D personality and poor health: a cross-sectional investigation. PLoS One 2017; 12: e0176014.

Conden E, Ekselius L, Aslund C. Type D personality is associated with sleep problems in adolescents. Results from a population-based cohort study of Swedish adolescents. J Psychosom Res 2013; 74: 290-295.

Kupper N, Pelle A, Denollet J. Association of type D personality with the autonomic and hemodynamic response to the cold pressor test. Psychophysiology 2013; 50: 1194-1201.

Kop WJ, Stein PK, Tracy RP, et al. Autonomic nervous system dysfunction and inflammation contribute to the increased cardiovascular mortality risk associated with depression. Psychosom Med 2010; 72: 626-635.

Jenkins CD, Stanton BA, Niemcryk SJ, et al. A scale for the estimation of sleep problems in clinical research. J Clin Epidemiol 1988; 41: 313-321.

Mommersteeg PM, Herr R, Bosch J, et al. Type D personality and metabolic syndrome in a 7-year prospective occupational cohort. J Psychosom Res 2011; 71: 357-363.

Juskiene A, Podlipskyte A, Bunevicius A, et al. Type-D personality and sleep quality in coronary artery disease patients with and without obstructive sleep apnea: mediating effects of anxiety and depression. Int J Behav Med 2018; 25: 171-182.

Burkauskas J, Brozaitiene J, Bunevicius A, et al. Association of depression, anxiety, and type-D personality with cognitive function in patients with coronary artery disease. Cogn Behav Neurol 2016; 29: 91-99.

Staniute M, Brozaitiene J, Burkauskas J, et al. Type-D personality, mental distress, social support and health-related quality of life in coronary artery disease patients with heart failure: a longitudinal observational study. Health Qual Life Outcomes 2015; 13: 1.

Prochaska JO, Velicer WF. The transtheoretical model of health behavior change. Am J Health Promot 1997; 12: $38-48$. 
Bandura A. Social foundations of thought and action. Englewood Cliffs, NJ, Prentice-Hall Inc., 1986

Richards D, Bartlett DJ, Wong K, et al. Increased adherence to CPAP with a group cognitive behavioral treatment intervention: a randomized trial. Sleep 2007; 30: 635-640.

Wohlgemuth WK, Chirinos DA, Domingo S, et al. Attempters, adherers, and non-adherers: latent profile analysis of CPAP use with correlates. Sleep Med 2015; 16: 336-342.

Clark NM, Becker MH Theoretical models and strategies for improving adherence and disease management. In: Shumaker SA, Schron EB, Ockene JK, et al., eds. The handbook of health behavior change. 2nd Edn. New York, NY, Springer Publishing Co, 1998; pp. 5-32.

Quine L, Rutter DR, Arnold L. Comparing the theory of planned behaviour and the health belief model: the example of safety helmet use among schoolboy cyclists. In: Abraham C ed. Understanding and changing health behaviour: from health beliefs to self-regulation. 2000; pp. 73-98.

Sage CE, Southcott A-M, Brown SL. The health belief model and compliance with CPAP treatment for obstructive sleep apnoea. Behav Change 2001; 18: 177-185.

Moran AM, Everhart DE, Davis CE, et al. Personality correlates of adherence with continuous positive airway pressure (CPAP). Sleep Breath 2011; 15: 687-694.

Carver CS, White TL. Behavioral inhibition, behavioral activation, and affective responses to impending reward and punishment: the BIS/BAS scales. J Person Soc Psychol 1994; 67: 319.

Copur AS, Erik Everhart D, Zhang C, et al. Effect of personality traits on adherence with positive airway pressure therapy in obstructive sleep apnea patients. Sleep Breath 2018; 22: 369-376.

Shahrabani S, Tzischinsky O, Givati G, et al. Factors affecting the intention and decision to be treated for obstructive sleep apnea disorder. Sleep Breath 2014; 18: 857-868.

Maschauer EL, Fairley DM, Riha RL. Does personality play a role in continuous positive airway pressure compliance? Breathe (Sheff) 2017; 13: 32-43.

Brostrom A, Stromberg A, Martensson J, et al. Association of type D personality to perceived side effects and adherence in CPAP-treated patients with OSAS. J Sleep Res 2007; 16: 439-447.

Gulati A, Ali M, Davies M, et al. A prospective observational study to evaluate the effect of social and personality factors on continuous positive airway pressure (CPAP) compliance in obstructive sleep apnoea syndrome. BMC Pulm Med 2017; 17: 56.

Sawyer AM, King TS, Hanlon A, et al. Risk assessment for CPAP nonadherence in adults with newly diagnosed obstructive sleep apnea: preliminary testing of the Index for Nonadherence to PAP (I-NAP). Sleep Breath 2014; 18: 875-883.

Hoy CJ, Vennelle M, Kingshott RN, et al. Can intensive support improve continuous positive airway pressure use in patients with the sleep apnea/hypopnea syndrome? Am J Respir Crit Care Med 1999; 159: 1096-1100.

Popescu G, Latham M, Allgar V, et al. Continuous positive airway pressure for sleep apnoea/hypopnoea syndrome: usefulness of a 2-week trial to identify factors associated with long term use. Thorax 2001; 56: 727-733. Hui DS, Chan JK, Choy DK, et al. Effects of augmented continuous positive airway pressure education and support on compliance and outcome in a Chinese population. Chest 2000; 117: 1410-1416.

Platón MJR, Sierra JE. Changes in psychopathological symptoms in sleep apnea patients after treatment with nasal continuous positive airway pressure. Int J Neurosci 1991; 62: 173-195.

Wozniak DR, Lasserson TJ, Smith I. Educational, supportive and behavioural interventions to improve usage of continuous positive airway pressure machines in adults with obstructive sleep apnoea. Cochrane Database Syst Rev 2014; 1: Cd007736.

Lai AYK, Fong DYT, Lam JCM, et al. The efficacy of a brief motivational enhancement education program on CPAP adherence in OSA: a randomized controlled trial. Chest 2014; 146: 600-610.

Cvengros JA, Rodriguez VM, Snyder S, et al. An adaptive treatment to improve positive airway pressure (PAP) adherence in patients with obstructive sleep apnea: a proof of concept trial. Behav Sleep Med 2017; 15: 345-360.

Chervin $\mathrm{RD}$, Theut $\mathrm{S}$, Bassetti $\mathrm{C}$, et al. Compliance with nasal CPAP can be improved by simple interventions. Sleep 1997; 20: 284-289.

Likar LL, Panciera TM, Erickson AD, et al. Group education sessions and compliance with nasal CPAP therapy. Chest 1997; 111: 1273-1277.

Aloia MS, Ilniczky N, Di Dio P, et al. Neuropsychological changes and treatment compliance in older adults with sleep apnea. J Psychosom Res 2003; 54: 71-76.

Stepnowsky CJ, Palau JJ, Gifford AL, et al. A self-management approach to improving continuous positive airway pressure adherence and outcomes. Behav Sleep Med 2007; 5: 131-146.

Almirall D, Nahum-Shani I, Sherwood NE, et al. Introduction to SMART designs for the development of adaptive interventions: with application to weight loss research. Transl Behav Med 2014; 4: 260-274.

Montesi L, El Ghoch M, Brodosi L, et al. Long-term weight loss maintenance for obesity: a multidisciplinary approach. Diabetes Metab Syndr Obes 2016; 9: 37-46.

Generali I, De Panfilis C. Personality traits and weight loss surgery outcome. Curr Obes Rep 2018; 7: 227-234.

Livhits M, Mercado C, Yermilov I, et al. Preoperative predictors of weight loss following bariatric surgery: systematic review. Obes Surg 2012; 22: 70-89.

Dave R, Calugi S, El Ghoch M. Are personality characteristics as measured by the temperament and character inventory (TCI) associated with obesity treatment outcomes? A systematic review. Curr Obes Rep 2018; 7: 27-36.

Sullivan S, Cloninger CR, Przybeck TR, et al. Personality characteristics in obesity and relationship with successful weight loss. Int J Obes (Lond) 2006; 31: 669-674.

Smith SS, Doyle G, Pascoe T, et al. Intention to exercise in patients with obstructive sleep apnea. J Clin Sleep Med 2007; 3: 689-694.

Igelstrom H, Martin C, Emtner M, et al. Physical activity in sleep apnea and obesity-personal incentives, challenges, and facilitators for success. Behav Sleep Med 2012; 10: 122-137.

Byrne S, Barry D, Petry NM. Predictors of weight loss success. Exercise versus dietary self-efficacy and treatment attendance. Appetite 2012; 58: 695-698.

Kerrigan SG, Clark M, Convertino A, et al. The association between previous success with weight loss through dietary change and success in a lifestyle modification program. J Behav Med 2018; 41: 152-159. 
Farinholt GN, Carr AD, Chang EJ, et al. A call to arms: obese men with more severe comorbid disease and underutilization of bariatric operations. Surg Endosc 2013; 27: 4556-4563.

111 Marin RS. Apathy: a neuropsychiatric syndrome. J Neuropsychiatry Clin Neurosci 1991; 3: 243-254.

112 Vernet C, Redolfi S, Attali V, et al. Residual sleepiness in obstructive sleep apnoea: phenotype and related symptoms. Eur Respir J 2011; 38: 98-105.

113 Desouza CV, Padala PR, Haynatzki G, et al. Role of apathy in the effectiveness of weight management programmes. Diabetes Obes Metab 2012; 14: 419-423.

114 Frakey LL, Salloway S, Buelow M, et al. A randomized, double-blind, placebo-controlled trial of modafinil for the treatment of apathy in individuals with mild-to-moderate Alzheimer's disease. J Clin Psychiatry 2012; 73: 796-801.

115 Ruthirakuhan MT, Herrmann N, Abraham EH, et al. Pharmacological interventions for apathy in Alzheimer's disease. Cochrane Database Syst Rev 2018; 5: Cd012197.

116 Chapman JL, Cayanan EA, Hoyos CM, et al. Does armodafinil improve driving task performance and weight loss in sleep apnea? A randomized trial. Am J Respir Crit Care Med 2018; 198: 941-950.

117 Funayama T, Ikeda Y, Tateno A, et al. Modafinil augments brain activation associated with reward anticipation in the nucleus accumbens. Psychopharmacology (Berl) 2014; 231: 3217-3228.

118 Young JW, Geyer MA. Action of modafinil: increased motivation via the dopamine transporter inhibition and D1 receptors? Biol Psychiatry 2010; 67: 784-787.

119 Dobrosielski DA, Patil SP. Weight loss and obstructive sleep apnea: what lies AHEAD? Sleep 2013; 36: 627-629.

120 Johansson K, Neovius M, Lagerros YT, et al. Effect of a very low energy diet on moderate and severe obstructive sleep apnoea in obese men: a randomised controlled trial. BMJ 2009; 339: b4609.

121 Kuna ST, Reboussin DM, Borradaile KE, et al. Long-term effect of weight loss on obstructive sleep apnea severity in obese patients with type 2 diabetes. Sleep 2013; 36: 641-649.

122 Foster GD, Borradaile KE, Sanders MH, et al. A randomized study on the effect of weight loss on obstructive sleep apnea among obese patients with type 2 diabetes: the sleep AHEAD study. Arch Intern Med 2009; 169: 1619-1626.

123 Tuomilehto HP, Seppa JM, Partinen MM, et al. Lifestyle intervention with weight reduction: first-line treatment in mild obstructive sleep apnea. Am J Respir Crit Care Med 2009; 179: 320-327.

124 Cayanan EA, Marshall NS, Hoyos CM, et al. Maintenance diets following rapid weight loss in obstructive sleep apnea: a pilot 1-year clinical trial. J Sleep Res 2018; 27: 244-251.

125 Burke LE, Ewing LJ, Ye L, et al. The SELF trial: a self-efficacy-based behavioral intervention trial for weight loss maintenance. Obesity (Silver Spring) 2015; 23: 2175-2182.

126 Behm-Morawitz E, Lewallen J, Choi G. A second chance at health: how a 3D virtual world can improve health self-efficacy for weight loss management among adults. Cyberpsychol Behav Soc Netw 2016; 19: 74-79. 